rendered useless. One innovation that may bear fruit would be the development of a case register of the homeless mentally ill. This would give such individuals a better chance of receiving some form of ongoing, co-ordinated care, wherever they might present. Further, such a register would facilitate the equitable distribution of any central funding which may be directed towards the health care of the homeless.

Department of Health (1989) Working for Patients. London: HMSO.

Fisher, N., Turner, S. \& PUGH, R. (1990) Homeless and mentally ill. Lancet, 14 April, 914-915.

Susser, E., Conover, S. \& Struening, E. L. (1989) Problems of epidemiologic method in assessing the type and extent of mental illness among homeless adults. Hospital and Community Psychiatry, 40, 261-265.

Tessler, R. \& DenNis, D. (1989) A Synthesis of NIMH-Funded Research Concerning Persons Who are Mentally IIl. Washington DC: National Institute of Mental Health.

The Maudsley Hospital

Nigel FiSHER

Denmark Hill

London SE5 8 AZ

\section{Screening of admissions to accident and emergency}

SIR: Bell et al (Journal, April 1991, 158, 554-557) confirm numerous previous reports that it is always possible in the general hospital for psychiatrists to locate some patients whose psychiatric disorders have not been diagnosed by physicians or surgeons and others who have been inappropriately referred. Does this matter?

Almost invariably in this kind of study, some identified patients, although psychiatrically disordered, will be judged unsuitable for psychiatric treatment or may decline it, or treatment may prove ineffective or even detrimental; others, after leaving hospital, may be treated by general practitioners or reach psychiatrists through various agencies. Unless a comparison is made between the outcome of similar groups of patients referred or not referred to psychiatrists it remains an open question as to how much the medical staff's failure to detect psychiatric morbidity really matters.

Every specialty has to accept some inappropriate referrals, but Drs Bell et al do have a point to make about the automatic referral of all overdose patients to psychiatrists in the Accident and Emergency Department at University College Hospital. This practice ceased to be mandatory in 1984 when the DHSS amended its recommendation that all selfpoisoned patients should be seen by psychiatrists. Medical staff, however, are unlikely to be sufficiently motivated to carry out an initial psychiatric assessment if psychiatrists - or indeed psychiatric nurses or social workers as Drs Bell et al propose - are always available to do the job for them.

There is in fact ample evidence that medical and accident service staff, given suitable training, are competent to assess suicidal risk in their overdose patients and to decide the need for psychiatric or social work referral (e.g. Waterhouse \& Platt, 1990). By gaining experience in making this assessment they may incidentally improve their ability to listen to the generality of their patients and to detect if they are psychiatrically unwell.
WATERHouse, J. \& Platt, S. (1990) General hospital admission in the management of parasuicide: a randomised controlled trial. British Journal of Psychiatry, 156, 236-242.

\section{R. GARDNER}

Addenbrooke's Hospital

Hills Road

Cambridge CB2 2QQ

\section{What's in a name?}

SIR: Changing names/titles is a frequently used device to signify real or imagined change and progress (e.g. Windscale becomes Sellafield, Mental Illness Service becomes Mental Health Service, etc.). Now Consultant Psychiatrist, at least in Dr Falloon's case, becomes "Consultant Physician (Mental Health)" (Rea et al, Journal, May 1991, 158, 642647). This title may be less daunting and less stigmatising among patients and even other medical colleagues, although it could cause confusion as psychiatrists are not, at least in popular understanding, physicians.

I rather like the historical "Mental Hygienist"; perhaps other psychiatrists have suggestions for a new name. Should we consult widely on this issue and change not only our titles but the name of the College?

\section{Royal Hallamshire Hospital \\ Glossop Road \\ Sheffield S10 2JF}

P. POWER-SMITH

\section{Anorexia nervosa in the elderly}

SIR: O'Shea (Journal, May 1991, 158, 716-717) questions the diagnosis of anorexia nervosa in the elderly lady we previously described (Journal, February $1991,158,286-287$ ), and suggests that an atypical affective disorder was a more likely explanation of her symptoms. This assertion we believe to be 
unfounded, as at no stage during our involvement with this lady did she demonstrate any affective symptoms whatsoever. Instead she demonstrated the typical features of anorexia nervosa, as defined by the DSM-III-R criteria, of severe weight loss, a typical distortion of body image, and a fear of becoming fat. Amenorrhoea was obviously not of relevance because of her advanced age. Although it is difficult to be certain of the exact circumstances surrounding her first episode fifty years beforehand, her account, confirmed by that of other family members, is strongly indicative of anorexia nervosa, and gives no cause to promote an alternative diagnosis.

Reports of eating disorders in the elderly are not restricted to that of Bernstein (1972), which O'Shea rightly questions. We have reviewed sixteen cases of anorexia nervosa or bulimia nervosa in the overfifties reported in the recent literature (Cosford \& Arnold, submitted), and found serious doubt regarding the diagnosis in only four. The remaining twelve demonstrated the typical psychopathology of an eating disorder, apart from the patient's age. Interestingly, half had arisen for the first time in later life, while the other half had initially arisen in the patient's youth, and they either remained persistently unwell for many years, or suffered a relapsing and remitting course, with remissions of up to fifty years in some cases.

It is our assertion that advanced age should not by itself rule out the diagnosis of anorexia nervosa, when the typical features are undoubtedly present, and there is no alternative major physical or psychiatric cause for the patient's symptoms.

BERNSTEIN, I. (1972) Anorexia nervosa. Ninety-four year old woman treated with electroshock. Minnesota Medicine, 55, 552-553.

Department of Psychiatry

St Mary's Hospital

London W2

Central Middlesex Hospital

Elaine Arnold

London NWI0

Buspirone-induced mania: possible interaction with disulfiram

SIR: McIvor \& Sinanan (Journal, January 1991, 158, 136-137) report the case of an alcoholic patient who developed mania when treated with buspirone $20 \mathrm{mg} /$ day and disulfiram $400 \mathrm{mg} /$ day. The authors attribute mania to buspirone treatment and suggest a possible mechanism consisting of facilitation of dopaminergic functions by this drug.
Although azapirones can have antidepressant effects (Robinson, 1989) and buspirone could cause mania by itself, we have reasons to believe that in this particular case the interaction between buspirone and disulfiram could cause mania. Drs McIvor \& Sinanan point out that no significant interaction has been reported with buspirone and disulfiram. However, both drugs can interact in at least two sites: (a) the brain, and (b) the liver.

(a) The brain: disulfiram inhibits dopamine betahydroxylase resulting in decreased brain norepinephrine levels and increased dopamine levels (Ciraulo \& Ciraulo, 1988). Through this mechanism disulfiram can enhance the facilitation of dopaminergic action by buspirone and contribute to the development of mania. In fact, the patient reported by Drs McIvor \& Sinanan required high-dose neuroleptic medication to control his symptoms, which concurs with the presence in the synaptic cleft of high concentration of dopamine (as could be expected with this mechanism). Furthermore, it has been observed that alcoholics with low cerebrospinal fluid dopamine beta-hydroxylase activity are more likely to develop dysphoric symptoms (Ciraulo \& Ciraulo, 1988). It could be argued that the patient took disulfiram only intermittently, which makes a possible buspironedisulfiram interaction less likely. However, two factors must be considered: (a) disulfiram is eliminated within three to five days and its principal metabolite (diethyldithiocarbamate) also inhibits dopamine beta-hydroxylase; (b) the patient was taking $400 \mathrm{mg} /$ day of disulfiram which is a high dose compared with the standard dose recommended to avoid adverse effects: $250 \mathrm{mg} /$ day (Kaplan \& Sadock, 1988).

(b) The liver: disulfiram is an inhibitor of the hepatic microsomal enzyme oxidase system (Ciraulo \& Ciraulo, 1988). As buspirone is eliminated by oxidative metabolism (Gammans et al, 1986), disulfiram could contribute to the elevation of plasma levels of buspirone by delaying its elimination.

In summary, by either or both of these mechanisms, disulfiram could have contributed, with buspirone, to the development of mania. Therefore we think that a possible buspirone-disulfiram interaction must be considered when buspirone is prescribed as a non-addictive anxiolytic in alcoholic patients on disulfiram.

Ciraulo, D. A. \& Ciraulo, A. M. (1988) Substance abuse. In Handbook of Clinical Psychopharmacology (eds J. P. Tupin, R. I. Shader \& D. S. Harnett), pp. 121-157. Northvale: Jason Aronson Inc.

Gammans, R. E., Mayol, R. F. \& LABUDDE, J. A. (1986) Metabolismo y disponibilidad de la buspirona. American Journal of Medicine (edición en español), 80 (suppl. 3B), 42-52.

KAPLAN, H. I. \& SADOCK, B. J. (1988) Synopsis of Psychiatry, Behavioural Sciences, Clinical Psychiatry. (5th edn). Baltimore: Williams \& Wilkins. 